Conclusion We found very high satisfaction with and loyalty to GCO among first-time users, indicating a successful service model from a client perspective. In addition to uptake and test outcomes, user experience is a key outcome for evaluation of online HIV/STI testing services.

\section{P2.28 ART - INDUCED NEPHROTOXICITY AND CHRONIC KIDNEY DISEASES AMONG AMBULATORY HIV - INFECTED PATIENTS WITH LOW BODY MASS INDEX IN BRAZZAVILLE, CONGO: INCIDENCE AND ASSOCIATED RISK FACTORS}

Martin Herbas Ekat. University Hospital of Brazzaville

10.1136/sextrans-2017-053264.204

Introduction To describe the incidence and risks factors of ART induced nephrotoxicity and chronic kidney disease (CKD) in HIV-1-infected adults with low body mass index $\left(<18.5 \mathrm{~kg} / \mathrm{m}^{2}\right)$.

Methods A retrospective cohort study at the Ambulatory Treatment Centre in Brazzaville, Congo. Patients with estimated glomerular filtration rate (eGFR) decrease by $25 \%$ compared to baseline or a $0.5 \mathrm{mg} / \mathrm{dL}$ increase in Serum creatinine ( $\mathrm{Scr}$ ) above baseline were classified as having nephrotoxicity, and $\mathrm{CKD}$ was defined as a value less than $60 \mathrm{ml} / \mathrm{min}$ per $1.73 \mathrm{~m}^{2}$. We used Cox proportional hazards regression models to determine factors associated with nephrotoxicity and CKD. Results Of 325 patients, $73.23 \%$ were women. Median values was: age: 37.55 years (IQR: 33.51-44.96), weight: $45 \mathrm{~kg}$ (IQR: 41-49), CD4 count: 137.5 cells/ $\mu 1$ (42 - 245). In the first 24 - months followup on ART incidence rate of nephrotoxicity and CKD was 27.95 and 7.44 per 100 person - years respectively. Multivariate analysis identified as a risk factor of nephrotoxicity, baseline haemoglobin below or equal $8 \mathrm{~g} / \mathrm{dL}$ $(\mathrm{aHR}=2.25 ; 95 \% \mathrm{CI}, 1.28-3.98 ; \mathrm{p}=0.005)$, eGFR between $60-80(\mathrm{aHR}=0.33 ; 95 \% \mathrm{CI}, 0.20-0.56 ; \mathrm{p}=0.001)$ and below $60 \mathrm{ml} / \mathrm{min} / 1.73 \mathrm{~m}^{2} \quad(\mathrm{aHR}=0.11 ; 95 \% \mathrm{CI}, 0.03-0.46 ; \mathrm{p}=0.003)$, and the use of tenofovir $(\mathrm{aHR}=1.51 ; 95 \% \mathrm{CI}, 1.01-2.26$; $\mathrm{p}=0.04)$. Each 10 year older age was associated with an increased risk of developing CKD $(\mathrm{aHR}=1.95$; 95\% CI, 1.2$3.17 ; \mathrm{p}=0.007)$.

Conclusion Incidence of nephrotoxicity and CKD were high. HIV positive patient with low BMI at baseline need close monitoring of their renal function when treated with tenofovir.

\section{P2.29 PERFORMANCE OF A CLINICAL PREDICTION SCORE FOR TARGETED CREATININE TESTING IN AFRICA}

Martin Herbas Ekat. University Hospital of Brazzaville, Republic of Congo

\subsection{6/sextrans-2017-053264.205}

Introduction in sub-saharian africa, in clinical practice only patients meeting the WHO criteria to start antiretroviral treatment undergone creatinine testing, by lack of reagents. Some patients with renal dysfunction are not diagnosed. Van Griensven et al. developed a clinical prediction score (CPS), to accurately identify Cambodian patients, with median baseline body weight of $49 \mathrm{~kg}$ (IQR: 43-55), who need a creatinine test before initiating antiretroviral therapy, based on the post-test probability of renal dysfunction. We evaluated the performance of this CPS for predicting patients at risk of kidney dysfunction (KD) in Brazzaville, Congo.

Methods The CPS includes following predictors: age (score +2 if $>40$ years), body weight (score +2 if $<45 \mathrm{~kg}$ ), and haemoglobin (score +1 if $>10 \mathrm{~g} / \mathrm{dL}$ ), the alternative CPS includes: age (score +2 if $>40$ years), body weight (score +2 if $<45 \mathrm{~kg}$ ), sex (score +1 if female), and WHO stage (score +1 if WHO stage III/IV). The overall test performance of the CPS was assessed by calculating the area under the receiver-operating characteristic (AUROC) curve. We defined KD as an estimated creatinine clearance based on the Cockcroft - Gault equation, by using two threshold, $<50$ (CG1) and $<60$ (CG2) $\mathrm{mL} / \mathrm{min}$. Results Among 545 patients, median values was: body weight $55 \mathrm{~kg}$ (IQR 48-63), age 38.87 years (33.18-46.21); 76 $(13.95 \%)$ and $142(26.06 \%)$ patients, respectively for CG1 and CG2, had KD. AUROC was 0.6183 (CPS) and 0.5815 (alternative CPS) for CG1 ( $\mathrm{p}=0.0541), 0.7140$ (CPS) and 0.6691 (alternative CPS) for CG2 $(\mathrm{p}=0.0016)$.

Conclusion Because the baseline body weight of African is high than in Cambodian patients, the CPS must be designed for African patients, it is a useful supplement to clinical judgment in the era of limited resources.

\section{P2.30 SURVEY OF ANTIMICROBIAL RESISTANCE IN CLINICAL NEISSERIA GONORRHOEAE ISOLATED OVER A PERIOD OF FOUR YEARS IN NAIROBI - KENYA}

${ }^{1}$ Meshack Juma Omolo, ${ }^{1}$ Lewa Pole, ${ }^{1}$ Isabella Mwangi, ${ }^{2}$ Joseph Kimani, ${ }^{1}$ Omu Anzala ${ }^{3}$ Jonathan Oloo, Wi, ${ }^{4}$ Teodora Elvira, ${ }^{5}$ Susanne Jacobsson, ${ }^{6}$ Magnus Unemo. ${ }^{1}$ Kavi-Institute of Clinical Research (UON), Nairobi, Kenya; ' 2 Sex Workers Outreach Program Clinic (SWOP), Nairobi, Kenya; ${ }^{3}$ Dept Medical Microbiology (UON), Nairobi, Kenya; ${ }^{4}$ World Health Organisation (WHO), Geneva, Switzerland; ${ }^{5}$ WORLD WHO Collaboration Centre for Gonorrhoea and Other ST/S Orebro University, Örebro, Sweden; ${ }^{6}$ WORLD WHO Collaboration Centre for Gonorrhoea and Other STIs Örebro University, Örebro, Sweden

\subsection{6/sextrans-2017-053264.206}

Introduction There is increasing urgency to document changing antimicrobial resistance (AMR) patterns of $N$. gonorrhoea (GC) in different parts of the world. High-level resistance to previously recommended quinolones is widespread and decreased susceptibility to the extended-spectrum (third-generation) cephalosporin. The surveillance for AMR in Kenya and the region was undertaken to determine the frequency and diversity of antimicrobial resistance of gonococcal isolates from Sex Workers Outreach Program (SWOP) Clinic.

Methods The survey tested 238 isolates over a period of 4 years from participants presenting with cervical/vaginal discharge. Samples collected were inoculated directly on modified Thayer martin media (MTM), transported to GASP Laboratories at KAVI-Institute of Clinical Research and identified by standard bacteriological procedures. Antibiotic susceptibility testing of $G C$ isolates was performed using diffusion gradient method. The MICs of penicillin, tetracycline, ciprofloxacin, spectinomycin, erythromycin, Azithromycin, cefixime and ceftriaxone were determined by the E-test method. The strains were defined as susceptible, intermediate and resistant using the WHO guidelines, all the findings were validates at WHO Collaborating Centre for Gonorrhoea and other STIs, Örebro University Hospital in Sweden.

Results 41 isolates in 2012,119 isolates in 2013, 24 isolates in 2014 and 54 isolates in 2015 showed 100\% susceptaility for cefixime, ceftriaxone and spectinomycin, with a mean susceptibility of $82 \%, 37.7 \%, 19.5 \%, 1.6 \%$ and $0 \%$ for azithromycin, 